\title{
Representaciones sociales de docentes en la modalidad b-learning desde una perspectiva sociocrítica
}

\section{Social representations of teachers in the b-learning modality from a socio-critical perspective}

\author{
PADILLA, José E. ${ }^{1}$ \\ SILVA, Wilmer H. ${ }^{2}$ \\ COLORADO, Paula ${ }^{3}$
}

\section{Resumen}

Este artículo considera las perspectivas conceptuales producto del análisis de las representaciones sociales que se configuran alrededor del rol del docente en la modalidad b-learning en educación superior. La consolidación de los resultados se desarrolló mediante la triangulación hermenéutica como proceso metodológico, donde se analizó la percepción que el docente tiene de sí desde una aproximación pedagógica sociocrítica. Se concluye que la configuración de las representaciones sociales del docente está mediada por una formación pedagógica e investigativa sociocrítica.

Palabras clave: educación a distancia, papel del docente, transferencia de conocimientos, investigación pedagógica

\begin{abstract}
This article considers the conceptual perspectives resulting from the analysis of the social representations that are configured around the role of the teacher in the b-learning modality in higher education. The consolidation of the results was developed through hermeneutic triangulation as a methodological process, where the teacher's perception of himself from a socio-critical pedagogical approach was analyzed. It is concluded that the configuration of the social representations of the teacher is mediated by a socio-critical pedagogical and investigative training.

key words: distance education, teaching role, Transfer of knowledge, pedagogical research
\end{abstract}

\section{Introducción}

El proyecto de investigación "Representaciones Sociales sobre el perfil del docente-tutor en la educación virtual de la Universidad Militar Nueva Granada", financiado por la Vicerrectoría de Investigaciones e identificado con el código INV-DIS-1754, tuvo como objetivo particular analizar tanto la perspectiva pedagógica que subyace al ejercicio docente dentro y fuera de las instituciones universitarias, como también, la perspectiva del docente en la modalidad b-learning como gestor del conocimiento. Se advirtió que convergen otras categorías nucleares

\footnotetext{
${ }^{1}$ Docente titular. Facultad de Estudios a Distancia. Universidad Militar Nueva Granada. eduardo.padilla@unimilitar.edu.co

2 Docente. Universidad de San Buenaventura. Universidad. wisilca@gmail.com

${ }^{3}$ Docente asociado. Departamento de Tecnologías del Conocimiento. Universidad Militar Nueva Granada. paula.colorado@unimilitar.edu.co
} 
como la formación pedagógica, la dimensión investigativa, la utilización y manejo desde la didáctica que permite las Tecnologías de Información y Comunicación (TIC)

Con respecto a la formación pedagógica, el objetivo del proyecto se concretó en estudiar el sustrato que fundamenta conceptual y epistemológicamente la configuración de las diversas representaciones sociales para la construcción del saber en todos los procesos de educación virtual e implementación de ambientes virtuales de aprendizaje, concretamente, en los contextos b-learning.

El análisis de las representaciones sociales tiene como sedimento, también, la pregunta por la formación investigativa que se da en las instituciones universitarias y el alcance que esta pueda tener. Situación que implica analizar aquellas en las que se da el ejercicio concreto de la docencia, aquellas convergencias, diferencias o distinciones entre la figura de docente como educador y como docente-investigador; lo cual se constituyó en otro objetivo de la investigación. De igual modo, dicho escenario da lugar a la comprensión del impacto del rol social que el docente tiene (padre, hijo, amigo u otro) en la configuración de su rol docente. Se trata así de indagar por aquellos márgenes de comprensión que se dan en torno a una posible jerarquización del rol social del docente o de la subordinación al rol profesional que este ejerce.

\subsection{Marco teórico}

\section{Blended learning}

La enseñanza al igual que los avances tecnológicos se ha ido transformado; los modelos de enseñanzaaprendizaje mediados por el uso de las TIC han puesto un referente en procura de la adaptación de nuevas herramientas para orientar los aprendizajes y lograr el conocimiento más allá de las fronteras espaciotemporales y es allí donde E-Learning, blended learning y mobile learning brindan una diversidad de interactividades para la articulación pedagógica (Bartolomé, García y Aguaded, 2018). Por ello, las tecnologías emergentes, el uso de dispositivos, las diferentes formas de acceso a la información y las redes sociales han facilitado la generación de nuevos modelos de enseñanza aprendizaje con miras a un movimiento global desde el enfoque pedagógico potenciando el quehacer docente y su implementación en la educación superior.

Es decir que el blended learning surgió como nuevo concepto para comprender los métodos de enseñanza aprendizaje, superando las barreras espacio temporales, ocasionando transformaciones en estas técnicas. La masificación de la conectividad en todo tipo de actividad y de ambiente social permite hoy entender que las TIC y el b-learning ofrecen potencialidades de implementación, para generar cambios estructurales a nivel formativo, donde juega un papel importante el rol del docente.

Esta combinación de métodos que brinda b-learning venía siendo desarrollado por aquellas primeras Instituciones de Educación superior (IES) que ofrecían programas en modalidad a distancia apoyados con tutorías presenciales, cuando todavía no eran tan accesibles las tecnologías digitales (García, 2018). Por tanto, el rol del docente ha sido fundamental y se ha venido fortaleciendo para el mundo actual, quien debe estar preparado en su disciplina, pero a la vez en el uso de las herramientas tecnológicas que le permitan aportar nuevo conocimiento a sus estudiantes, teniendo en cuenta que debe articular, integrar, armonizar las actividades, los mecanismos, las tecnologías adecuadas para suplir las necesidades de enseñanza aprendizaje y así lograr el equilibrio posible a nivel curricular.

La combinación de las TIC y sus múltiples aplicaciones para la formación agrega actividades a las ya desarrolladas tradicionalmente por los docentes, fortaleciendo su labor e impulsándolo para proyectar nuevos escenarios pedagógicos. Por lo anterior, Duarte, Guzmán, y Yot (2018) aseguran que,

cada vez aparecen más estudios que demuestran la importancia que tiene el desarrollo profesional docente $y$ el liderazgo en los procesos formativos y organizativos que se producen en contextos 
educativos, ejerciendo una poderosa influencia en la planificación, desarrollo y evaluación, en la eficacia de las tareas técnicas propias de la gestión y en la calidad del sistema relacional del mismo (p.156).

Este modelo de formación contribuye con múltiples opciones para el desarrollo profesional y académico en los campos de la investigación, trabajo colaborativo, participación, resolución de conflictos y liderazgo, entre otros aspectos, que son fundamentales al interior de las instituciones acreditada en alta calidad.

\section{Las representaciones sociales en los contextos b-learning}

De acuerdo con el enfoque de Moscovici y Jodelet (1989), citados por Arias y Moya (2015), las representaciones sociales se concretan en un área de investigación que trata de comprender la forma en la que se constituye el significado de un objeto o un fenómeno, cómo los actores sociales interpretan el mundo social, las relaciones sociales que devienen sobre la base de las representaciones elaboradas, y cómo estas representaciones están integradas en el sistema cognitivo de los sujetos sociales. La representación tiene origen en el colectivo y es compartida por los individuos en sus grupos sociales de referencia, lo que constituye construcciones colectivas que definen la realidad social.

Este tipo particular de conocimiento, cuya ocupación es la de desarrollar y comprender las conductas y la interrelación entre los individuos, denota que la representación social deviene, asimismo, como un corpus de conocimiento organizado. Específicamente, es un complejo entramado de actividades psíquicas a través de las cuales el sujeto hace comprensible la realidad social y física, cuya finalidad es formar parte de un grupo o entrar en un intercambio de relaciones entre los sujetos. En ese sentido, haciendo una ampliación conceptual, las representaciones sociales son construcciones cognitivas compartidas en la interacción social cotidiana que ofrecen a las personas un sentido común de entendimiento.

El entorno educativo es un espacio lleno de las relaciones de interacción entre los actores involucrados. En cuanto a este escenario, se encuentran las representaciones sociales para la comprensión de la educación y la importancia de grupos armonizados de significados sociales en el proceso educativo y sus interacciones, que requieren una interpretación de la escuela y no simplemente una reproducción de la realidad escolar (Capelari, 2016).

Por otro lado, las representaciones sociales también aluden a la forma como se apropia el conocimiento en una comunidad o grupo particular, como lo mencionan Torres y Romero (2018) al sostener que la heterogeneidad de un equipo de aprendices rompe con la posibilidad de aprender como un estándar, porque pone de relieve la forma en que cada individuo se orienta por sus intereses concretos. En la educación b-learning esto lleva a una fuerte consideración significativa del trabajo compartido y colaborativo. Según Visbal (2020) el significado cambia con el proceso interpretativo que se desarrolla a través de la interacción social en espacios virtuales.

De igual manera, desde la posición teórica de las representaciones sociales es posible señalar que cada maestro tutor es parte de un grupo profesional que construye sus puntos de vista durante la interacción con el entorno social, y que ésta está más allá de constituirse en una recepción o configuración de imágenes, ideas o puntos de vista en común. Se trata más bien de un movimiento personal y colectivo en el que el sujeto también se constituye como parte de una comunidad.

\section{El rol docente desde la pedagogía sociocrítica}

Puede indicarse que la educación virtual y el modelo b-learning se posicionan en la educación superior como una alternativa práctica de formación, por lo que es importante definir el rol que el maestro está desempeñando en estos nuevos escenarios en los que interactúa; pues a través de la información suministrada por los participantes mediante las entrevistas, se habla de la necesidad de un cambio en el rol docente, es decir, estos retos e innovaciones requieren que el docente se asuma como un agente reflexivo, autocrítico y analítico. 
De acuerdo con Mclaren (2015), los docentes que toman el rol de 'intelectuales transformadores consideran a los educandos como actores críticos, que difieren sobre la manera en que se produce y distribuye el conocimiento, utilizando el diálogo para lograr un conocimiento representativo, crítico y, finalmente, emancipatorio.

Por consiguiente, cuando se habla de un docente reflexivo se hace referencia a aquel que se cuestiona frente a su quehacer, porque más allá de que las clases sean presenciales o en el aula virtual, aquí el desafío es que se vincule la acción con la reflexión en acto pedagógico y la propia práctica, permitiéndole al educador la construcción de un rol docente transformador, rol que se relacione con los cambios de la comunidad y la cultura; como plantea McLaren (2015), los maestros deben libremente articular y utilizar un discurso crítico para constituir las practicas educativas fundamentadas en un espacio público para capacitar individual y socialmente dentro y fuera de los planteles. Probablemente este panorama enaltece a los maestros como ilustrados y detractores sociales y también relaciona su práctica con los compromisos formativos que generan un ambiente de aprendizaje crítico, social y democráticamente posible.

De igual forma, no se puede asumir la modalidad b-learning solamente como una mediación que facilite el proceso de formación y que proporcione comunicación con los estudiantes, porque más allá de aquello, se trata de una transformación a nivel educativo que integra factores políticos, económicos, sociales e históricos; y como tal, permite un ambiente de análisis y reflexión crítica sobre la incidencia de éste en la sociedad. Por ello, las acciones de la comunidad académica deben estar dirigidas a garantizar coherencia e integridad, no sólo de saberes y contenidos, sino desde la función social que cumple.

Por su parte, Giroux (2003) habla del papel del docente, no como aquel profesional de la educación, sino que se refiere al maestro que desempeña su labor como creador, movilizador y transmisor de la cultura. Señala:

Esto no significa convocar a los docentes a adherir a algún ideal abstracto que los apartes de la vida cotidiana o los convierta en profetas de la perfección y la certeza; al contrario, es convocarlos a ejercer la crítica social no como personas ajenas, sino como intelectuales públicos que abordan las cuestiones sociales y políticas de su barrio, su nación y el mundo en general (p. 313).

\section{Metodología}

Se acudió al paradigma interpretativo comprensivo para analizar la dimensión representacional de los profesores con relación a su desempeño en las modalidades de aprendizaje virtual y b-Learning; haciendo uso del enfoque cualitativo y mediante el método de comparación constante $\mathrm{MMC}$, se procedió a la aplicación de entrevistas semiestructuradas para la recolección de la información tomando como referente los aspectos conocidos entre los docentes de la Facultad de Estudios a Distancia de la Universidad Militar Nueva Granada. La población la constituyen docentes de educación superior de pregrado y posgrado de diferentes universidades nacionales e internacionales, seleccionados aleatoriamente, teniendo en cuenta que trabajen la modalidad b-learning. Lo anterior con el objeto de estudiar nuevas facetas teóricas que permitan comprender y proyectar esta modalidad de manera acertada.

La población informante estuvo integrada por quince docentes de educación superior (diez nacionales y cinco internacionales), con quienes se desarrolló el instrumento del proyecto. Para esto se aprovechó un evento académico de estudio en torno a los procesos educativos b-learning de carácter internacional, desarrollado en la ciudad de Medellín, Colombia. Con relación a la entrevista semiestructurada y la recolección de datos; para garantizar la fiabilidad de la información, se recurrió a la estrategia de videograbación de un diálogo espontáneo con los docentes. 
Luego de la recolección de datos, se procedió a su sistematización haciendo uso del Software ATLAS.ti elaborando categorías y códigos, a partir de elementos conceptuales determinados en el proceso investigativo. En ese sentido, se aborda el estudio desde la hermenéutica como método, fundamentalmente en la búsqueda de la ilustración al entendimiento (Ricoeur, 2017), como mecanismo para caracterizar y cimentar nuevo conocimiento.

Asimismo, se acudió a la triangulación hermenéutica dado que allí convergen varios factores que permiten una interpretación clara y de esta forma construir la teoría, en el entendido que el hecho de triangular facilita la articulación entre los referentes teóricos y la indagación (Padilla y Silva, 2017). En tal sentido Cisterna (2005) citado por los mismos autores considera que,

luego de reunir la información obtenida en la investigación, se cruza la información proporcionada por los informantes, estableciendo relaciones de comparación por categorías, lo que determina las conclusiones categoriales para, finalmente, cruzar las conclusiones con el marco teórico; y de este modo, una vez realizada la triangulación, obtenemos lo que se denomina resultados de la investigación (p.163).

Se toma como referente teórico el enfoque de la pedagogía sociocrítica desde Henry Giroux y Peter McLaren y la conceptualización que hace Moscovici de las representaciones sociales.

El logro último del proceso fue la consolidación de una de las unidades hermenéutica de comprensión o, vista de otra forma, la conformación de elementos discursivos tendientes a otros de comprensión del fenómeno estudiado.

\section{Resultados}

En el desarrollo de la educación virtual, el docente universitario se ha visto enfrentado al desafío de la innovación en su práctica educativa, apoyado en este proceso por las tecnologías y el despliegue de nuevas metodologías. Asimismo, este cambio se ha dado en respuesta a las necesidades de la población a la que se le dificulta la accesibilidad al proceso educativo presencial.

B-learning es un modelo por medio del cual los tutores pueden impartir sus temáticas a través del ambiente virtual; combinando la presencialidad con el rol del tutor de educación a distancia, es decir, que esta modalidad combina las prácticas presenciales con aquellas en las que se utilizan las TIC para mediar el conocimiento (Rodríguez et al. 2017)

Por otra parte, en este proceso los profesores devienen como difusores no sólo del saber - fenómeno diverso e inmediatos en el entorno educativo-, también, se configuran a sí mismos como un grupo social generador de representaciones sociales sobre la tecnología; en la medida en que es parte de su quehacer, "familiarizar» a sus colegas y estudiantes en el sentido y función de los procesos b-learning.

Es precisamente el objetivo de este artículo mostrar la definición del rol del docente en el modelo b-learning y las representaciones sociales que alrededor de éste se producen. Así, los aportes de la pedagogía crítica en esta perspectiva son importantes en la medida en que brindan un análisis de la influencia de los aspectos sociales, políticos, económicos y culturales frente al papel del maestro en la educación superior y cómo éste se asume en la incorporación de nuevas metodologías, siendo un actor social que puede generar transformaciones en los procesos pedagógicos.

De forma precisa, el estudio permitió inferir que se presentan las siguientes representaciones sociales de los docentes: gestor del saber, educador virtual, actor social y político. Desde el enfoque sociocrítico planteado en el estudio, se concluye que la representación del docente como un «intelectual transformador» ayudaría a sedimentar las representaciones previamente mencionadas acerca del docente. De este aspecto se dará cuenta a continuación. 


\subsection{El docente como gestor del saber en contextos B-Learning}

Los docentes entrevistados expresaron que las acciones que desarrollan las IES, con el fin de lograr un cambio del rol del docente tradicional, están dirigidas a capacitarlos en el uso de las tecnologías como apoyo a sus clases presenciales, ya que introducir en su práctica técnicas pedagogías y materiales acordes a estas innovaciones, se considera una transformación de su curso a modalidad b- learning. En este contexto, más allá del uso de las tecnologías, sus prácticas les movilizan como gestores del saber o del conocimiento. En la tabla 1 se muestran algunas de las voces de los entrevistados.

Tabla 1

Voz del informante como gestor del saber

\begin{tabular}{lc}
\hline \multicolumn{1}{c}{ Voz informante } & $\begin{array}{c}\text { Unidad discursiva } \\
\text { emergente }\end{array}$ \\
\hline «El rol del docente debe ser de gestor de conocimiento, siendo quien a & \\
través de las herramientas tecnológicas y de la virtualidad, potencia el \\
aprendizaje de sus estudiantes, manteniendo una adecuada interacción \\
comunicativa que facilita el proceso de enseñanza». \\
«En la universidad, el profesor con formación en tecnologías y uso de \\
herramientas virtuales es percibido con mayor respeto pues posee un \\
mayor conocimiento del tema». \\
«Se cree que el profesor de educación virtual tiene menos trabajo que el \\
de educación presencial, y este último es más valorado por un mejor \\
manejo en su área de conocimiento».
\end{tabular}

Fuente. Elaboración propia

Entonces, si bien es cierto que el docente debe replantear la enseñanza para la comunidad en constante cambio, también es cierto que debe cuestionarla e interrogarla antes de incorporarla a sus prácticas; asumiendo un rol de garante para que se den las condiciones necesarias en la construcción del saber al servicio de los estudiantes antes que a los intereses del capital y el mercado. Por lo tanto, como afirma Mclaren (2015), "El desafío para los maestros es reconocer e intentar transformar esos rasgos antidemocráticos y opresivos del control hegemónico que frecuentemente estructuran la existencia diaria de las aulas de modos no transparentes" (p. 301).

Estas acciones que las universidades están desarrollando para implementar el uso de las tecnologías continúan atadas a factores económicos, puesto que la aplicación de las normativas vigentes en la educación impide que las universidades sean autónomas, perjudicando de algún modo la democratización del saber. De acuerdo con Giroux (2001), las instituciones de educación superior están atravesadas por una cultura empresarial desde sus discursos y prácticas, generando una subordinación de la educación a las dinámicas del mercado. El autor define la cultura empresarial como:

Conjunto de fuerzas ideológicas e institucionales que, de una manera política y pedagógica, promueve tanto la dirección de la vida organizativa gubernamental a través del control por parte de los directivos, como el surgimiento de trabajadores dóciles, consumidores a políticos y ciudadanos pasivos (p. 47)

La educación superior debe permitir la integración y complementariedad entre lo que sucede en el contexto social, político, cultural y económico, y lo que ocurre en la escuela, pues no son temas aislados. El compromiso de la universidad consiste en la formación de seres humanos y no debe estar atada a intereses particulares y económicos; formando desde la autonomía y hacia la construcción de sujetos políticos con pensamiento crítico; generando procesos de reflexión y divergencia frente a la institucionalidad; capaces de defender no sólo sus derechos particulares, sino los intereses colectivos a través del reconocimiento y la interacción con el otro. Es decir, actores sociales que participan y contribuyen a transformación de la realidad en la cual se desenvuelven. 
Por esta razón, todas las acciones orientadas a impulsar la propuesta pedagógica de la modalidad b-learning deben articular sociedad, cultura y escuela; planteando nuevos roles para el docente que permita la democratización del saber, como lo plantea Giroux (2003):

Se trata de una posición marcada por un coraje moral y una crítica que no requiere que los educadores se aparten de la sociedad a la manera del docente "objetivo", sino que se distancien de las relaciones de poder que sojuzgan, oprimen y degradan a otros seres humanos. Los docentes deben encarar la crítica desde adentro, desarrollar prácticas pedagógicas que realcen no solo las posibilidades de la conciencia crítica, sino también las posibilidades de la acción transformadora (p. 213).

La modalidad b-learning es una alternativa útil para la población, ya que, al combinar entornos virtuales en los procesos de enseñanza, soluciona algunas limitaciones en cuanto a ubicación geográfica y tiempo. Sin embargo, no se reflejan consensos entre la comunidad académica de las universidades, puesto que cada institución le da un significado y una estructura diferente de acuerdo con sus necesidades y demandas. De esta manera, dicha modalidad se convierte sólo en un modelo económico desconociendo consideraciones educativas contextuales, lo que - bajo el paradigma del aprendizaje en la era digital, hacia la sociedad del conocimiento- disfraza sus verdaderos objetivos.

En consecuencia, el uso de herramientas virtuales se está planteando en términos de eficiencia y competitividad. De ahí que lo importante sea reducir costos y que erróneamente se dé por sentado que, si se usan ambientes tecnológicos en las clases o un software en el computador, se producen cambios en el proceso pedagógico, ignorando que continúan siendo los mismos procesos que en una clase presencial. A pesar de esto, la universidad y la sociedad privilegia al docente que se capacita en el uso de las TIC, ya que es visto como alguien que ingresa a la nueva era digital y se posiciona como un gestor de conocimiento. porque sus clases están mediadas por la tecnología y el uso de herramientas online o virtuales.

De acuerdo con la información suministrada por los participantes, es importante advertir un enorme riesgo. Se trata de que el docente como gestor del conocimiento, lo produce o reproduce a sus estudiantes, con la salvedad de que este maestro tiene una formación en TIC, pero aquí no se habla de un proceso de reflexión en cuanto a estas mediaciones, pues sólo constituyen una metodología más que han tenido que introducir en sus clases, y que favorecen el sistema dominante. De este modo, los maestros, deberían explorar el conocimiento con el ánimo de lograr una comprensión específica de cómo el estudiante es de hecho construido. Los conocimientos adquiridos en las aulas deberían ser fundamentales en la participación social del educando y la solución de problemáticas de su diario vivir, antes de ser simplemente un banco de valores del pragmatismo de los negocios (McLaren, 2015). Por esto, este rol del docente como gestor del conocimiento debe implicar una relación dialógica entre estudiante y maestro, en la cual se reconozcan como sujetos de aprendizaje, pues esta interacción posibilita al otro resignificar el saber.

El estudiante participa activamente en las aulas virtuales a través de foros, chats, "subiendo" informes, presentando evaluaciones o usando las bases de datos, entre otras. La inquietud aparece frente a si esta serie de actividades favorecen una actitud crítica del estudiante y le permiten plantear soluciones a problemas de la realidad; además, si estas dinámicas promueven el trabajo colectivo donde pueda existir un diálogo de saberes, ya que el conocimiento se construye con los otros.

Como lo afirma Mclaren (2015), el conocimiento está construido de acuerdo con la sociedad, es decir, se rige por consentimiento de las personas que sostienen relaciones sociales específicas y que viven en circunstancias particulares en el tiempo. Esto significa que el saber está social y simbólicamente construido a partir de la interacción social con los otros, lo que lo materializa como producto del contexto, las costumbres, la cultura y la historia. 


\subsection{Educador virtual vs. Educador presencial}

En la actualidad, surge el debate en torno a la educación y sus aportes en la sociedad, así como el rol que el docente desempeña en estas transformaciones sociales, por lo tanto, es importante caracterizar la imagen que se ha construido del modelo de formación b-learning y la comprensión del maestro que media sus clases con herramientas virtuales. En general, la concepción que se tiene es la de un «educador virtual» que se antepone a la de «educador presencial». En la tabla 2 se recogen algunas de las voces informantes al respecto.

Son variadas las percepciones que se tienen en torno a la educación virtual y el rol que el docente desempeña en entornos de virtuales, pues se dan lecturas de esta realidad no sólo desde la subjetividad, sino desde la mirada que los grupos sociales tienen frente a estas innovaciones en la educación superior, es decir, conforme lo señala Piñero (2008), citado por Higuita (2020),

La representación social constituye una forma de pensamiento social en virtud de que surge en un contexto de intercambios cotidianos de pensamientos y acciones sociales entre los agentes de un grupo social; por esta razón, [...] refleja la diversidad de los agentes y la pluralidad de sus construcciones simbólicas (p. 75).

Tabla 2

Voz del informante como educador virtual

\begin{tabular}{lc}
\hline \multicolumn{1}{c}{ Voz informante } & $\begin{array}{c}\text { Unidad discursiva } \\
\text { emergente }\end{array}$ \\
\hline «En la universidad el profesor con formación en tecnologías y uso de & \\
herramientas virtuales es percibido con mayor respeto pues posee un & \\
mayor conocimiento del tema». & Educador virtual \\
«Se cree que el profesor de educación virtual tiene menos trabajo que el \\
de educación presencial, y este último es más valorado por un mejor \\
manejo en su área de conocimiento ».
\end{tabular}

Fuente. Elaboración propia

Los datos proporcionados por los docentes entrevistados permiten evidenciar los imaginarios que se han construido en torno al docente en contextos b-learning, que toman como punto de partida un confrontación entre los modelos de educación virtual vs. educación presencial; existiendo para ellos una diferencia marcada en el rol que desarrolla el docente en cada una de estas modalidades dado que se cree que el docente que media sus clases con herramientas virtuales no posee la experticia en la disciplina impartida, pues al no estar cara a cara con sus estudiantes posee menor dominio de los temas; además -como emerge de la información obtenida- requiere de menos tiempo para preparar y desarrollar sus clases. Por el contrario, el docente presencial es visto con mayor respeto y tiene un mayor estatus en cuanto a los conocimientos que posee en su disciplina, no obstante, los estudiantes les exigen el uso de herramientas tecnológicas.

Según los participantes, la sociedad privilegia la educación presencial, ya que ésta cuenta con un requisito fundamental para el aprendizaje y es el hecho de que profesor y estudiante se encuentren en el mismo espacio y tiempo; en consecuencia, la educación virtual pierde credibilidad y seriedad, pues se cree que allí no existe interacción y comunicación entre sus actores, ya que la misma palabra «virtualidad» es concebida como algo irreal, lo que genera desconfianza por no ser tangible.

Sin embargo, la educación virtual en ningún momento anula el contacto con la realidad, sino que hace parte importante de ella, incluso, permite formas de interacción que no son usadas en la educación presencial, enriqueciendo de esta manera los procesos de comunicación gracias a las tecnologías usadas. Debido a que en este escenario convergen sujetos con diferentes propósitos que se unen con un fin común, esto es, la formación mediada por los recursos tecnológicos, para constituirse en sujetos productivos y en ciudadanos críticos y activos. 
Por lo tanto, el debate debe girar en torno a crear las condiciones necesarias, tanto pedagógicas, didácticas y tecnológicas, para la emergencia de nuevas modalidades diferentes a la presencial, debido a que estas permiten un mayor sentido y alcance en la sociedad de estas innovaciones educativas.

\subsection{El docente como un actor social y político}

La modalidad b-learning es percibida, asimismo, como una oportunidad para que las personas que poseen dificultades para acceder a la educación presencial, ya sea por dificultades de tiempo o de distancia, puedan lograrlo. Esto cuestiona aquella falsa comprensión según la cual es una modalidad de educación destinada exclusivamente a personas de bajos recursos, y como tal, no cuenta con todas las condiciones para ser considerada parte de la educación formal. Por ello, resulta imperativo que los docentes reconozcan que la educación en una sociedad burguesa no es una educación 'libre' y 'universal' y controviertan en relación con que la educación puede ser igual sin importar las clases sociales (McLaren, 2015).

Esta perspectiva social de la educación virtual es alimentada por la concepción de que los docentes en dicha modalidad se han ido configurando como actores sociales o como promotores de relaciones políticas desde la educación. En la tabla 3 se muestran algunas de las voces informantes que afirman tal perspectiva:

Tabla 3

Voz del informante como actor social

\begin{tabular}{lc}
\hline \multicolumn{1}{c}{ Voz informante } & $\begin{array}{c}\text { Unidad discursiva } \\
\text { emergente }\end{array}$ \\
\hline «El docente debe ejercer un rol en el que mínimo sepa comunicarse con \\
el uso de las tecnologías, tiene que aprender a plantear espacios \\
virtuales, a trabajar en equipo con los otros docentes para definir y \\
diseñar ambientes virtuales y saber preparar al estudiante en uso de las \\
tecnologías».
\end{tabular}

Fuente. Elaboración propia

Por lo tanto, se sostiene equívocamente que los contenidos son menos rigurosos y que los egresados bajo esta modalidad tendrán menos oportunidades en el mundo laboral; a esto se suma que las IES en el afán de cobertura: ante la necesidad de saldar la demanda y buscando ser competentes en el mercado laboral, ofrecen esta modalidad sin tener en cuenta los procesos pedagógicos que implica. Al respecto Mclaren (2015) precisa que, las escuelas han trabajado bajo el esquema de comercialización de la educación por estratos, lo cual profundiza la desigualdad cultural que degrada las relaciones sociales democráticas por la competitividad y el etnocentrismo cultural.

La realidad social exige que se traspongan estas consideraciones y que se promueva y facilite la comprensión de los docentes, especialmente en contextos b-learning, como sujetos políticos 'empoderado' de su papel de intelectuales públicos, como lo denomina Giroux (2018a), promoviendo estudiantes activos y críticos que se opongan a los discursos empresariales, enseñando a sus estudiantes el lenguaje de la crítica y de la responsabilidad social hacia condiciones más igualitarias; usando el lenguaje de la esperanza donde sea posible un cambio en las estructuras actuales del sistema y un docente como agente cultural que moviliza su realidad en beneficio de la justicia social y la democratización de la educación.

Sin embargo, aunque en las entrevistas se insiste en el rol del docente como gestor del conocimiento, se genera la probabilidad de que el docente se posicione o desempeñe su quehacer en términos de eficiencia y calidad, o como lo llama Giroux (2017), pedagogías gestionarías, las cuales apartan a los profesores de los procesos de 
razonamiento, pues no permiten participación y autonomía en el diseño, implementación y evaluación de sus clases; asimismo, no se tienen en cuenta las metodologías adecuadas para los entornos sociales en los cuales se desarrollan los procesos de formación.

Es importante que los docentes vuelvan a confiar en el ejercicio de su rol, considerándose como actores sociales y políticos que con sus prácticas generan transformaciones, para lo cual es necesario que se actualicen permanentemente en su área de conocimiento y accedan a formarse en estrategias de pedagogía virtual. Como muchos indican, aquello implica de parte de las instituciones universitarias, que estos esfuerzos también se vean reflejados en un reconocimiento salarial consecuente con el tiempo dedicado a estas prácticas.

Asimismo, que los grupos de estudiantes no sean tan numerosos permitiendo una mejor comunicación con el docente y de esta manera usar estas estrategias y herramientas virtuales hacia una educación crítica y reflexiva frente al conocimiento y estado actual de la sociedad. Por ello, como lo indica Moscovici (1978) citado por Lobato (2011), las representaciones sociales circulan, se insertan y se materializan a través de un discurso, un gesto, un encuentro, en general, en cada contexto cotidiano; y esto es posible si las condiciones son facilitadas por las instituciones.

Como resultado, el docente debe establecer su papel y función social como intelectual transformador, que logre que lo pedagógico sea más político, puesto que también es tarea de la universidad introducir la esfera política en la formación de sujetos que analicen y cuestionen las relaciones de poder en beneficio de una democratización justa. Además, que lo político se sirva de pedagogías permitiendo que los estudiantes participen en los procesos de toma de decisiones colectivas y que el educador se asuma como sujeto político que inste por el logro de un bien común (Giroux, 2018b).

\section{Conclusiones}

Este proyecto permitió concluir que la modalidad b-learning en educación no puede darse al margen de los esfuerzos que apuntan hacia el fortalecimiento de la identidad y la resignificación social de la profesión docente, teniendo en cuenta las dimensiones históricas, económicas, culturales y políticas en las que desarrolla su quehacer. Estas inquietudes deben estar relacionadas con sus prácticas y con una actitud reflexiva frente a las nuevas metodologías, en vista que los docentes que se están formando en TIC y en el diseño y uso de entornos virtuales se reconocen como protagonistas, no sólo del proceso educativo, sino, de transformación social. Agentes que a través de la investigación promueven el diálogo de saberes, las experiencias de aprendizaje y están inmersos en diferentes contextos educativos, reconociendo el sentido sociopolítico de la educación.

Por otra parte, se evidenció que la modalidad b-learning es la oportunidad para acceder a la educación superior rompiendo con estándares sociales en los que sólo unos pocos pueden acceder, ya que la virtualidad permite llegar a más personas, garantizando el acompañamiento y la formación permanente de un tutor o docente. Además, permite que los contenidos y actividades pedagógicas, de los cuales los estudiantes hacen uso por medio de una plataforma virtual, sean pensados en beneficio de una sociedad autónoma, democrática y justa.

Con todo, el proyecto de investigación permitió establecer que la figura sociocrítica del docente como un «intelectual transformador» ayuda a clarificar el sentido de las representaciones sociales que se han construido sobre el docente en la modalidad b-learning. Así, desde una perspectiva crítica, se resignifica la educación superior y la incidencia de las nuevas modalidades educativas, ante la transformación de la sociedad, que requiere que se formen ciudadanos con pensamiento crítico y divergente frente a la institucionalidad. De modo que sus aportes y su participación tiendan a movilizar y transformar su realidad, encontrando en estas nuevas formas de enseñar o mediar la construcción del saber, la posibilidad de brindar a los estudiantes las herramientas para formarse como sujetos sociales y políticos. 


\section{Referencias bibliográficas}

Arias, M., \& Moya, P. (2015). La relación entre las teorías de las representaciones sociales y la importancia de su estudio en los docentes universitarios. Investigación, Desarrollo e Innovación, 6(1), 61-71. Obtenido de https://revistas.uptc.edu.co/index.php/investigacion_duitama/article/view/4049/3490

Bartolomé, A., García, R., \& Aguaded, I. (2018). Blended learning: panorama y perspectivas. Revista Iberoamiericana de Educación, 21(1), 33-56. doi:http://dx.doi.org/10.5944/ried.21.1.18842

Capelari, M. (2016). El rol del tutor en la Universidad: Configuraciones, significados y prácticas. Sb editorial.

Duarte, A., Guzmán, M., \& Yot, C. (2018). Aportaciones de la formación blended learning al desarrollo profesional docente. Revista Iberoamericana de Educación a Distancia, 21(1), 155-174. doi:http://dx.doi.org/10.5944/ried.21.1.19013

García, L. (2018). Blended learning y la convergencia entre la educación presencial y a distancia. Revista Iberoamericana de Educación a Distancia, 21(1), 9-22. doi:http://dx.doi.org/10.5944/ried.21.1.19683

Giroux, H. (2001). Cultura, política y práctica educativa. New York: Grao.

Giroux, H. (2003). Pedagogía y política de la esperanza. España: Amorrortu Editores.

Giroux, H. (2017). Rethinking Higher Education in a time of Tyranny? Revista Runae, 2, 13-29.

Giroux, H. (2018a). Pedagogía crítica para tiempos difíciles. España: Editorial Mapas Colectivos.

Giroux, H. (2018b). Por qué importan los docentes en tiempos oscuros. Revista de Educación, 13, 13-19. Obtenido de https://fh.mdp.edu.ar/revistas/index.php/r_educ/article/view/2735/2715

Higuita, C. (2020). Representaciones, usos y construcciones sociales de la lengua escrita: otras literacidades. Cuadernos pedagógicos, 22(29), 71-77. Obtenido de https://revistas.udea.edu.co/index.php/cp/article/view/341882

Lobato, A. (2011). Reseña del libro A construção do objeto de pesquisa em representações sociais, del profesor Celso Pereira de Sá. Revista Internacional de Investigación en Educación, 3(6), 461-464. Obtenido de https://www.redalyc.org/pdf/2810/281021734014.pdf

McLaren, P. (2015). Life in Schools: An Introduction to Critical Pedagogy in the Foundations of Education (Sixth Edition ed.). New York: Paradigm Publishers.

Padilla, J., \& Silva, W. (2017). Impacto de las TIC en las representaciones sociales de los docentes en la modalidad b-learning. Civilizar: Ciencias Sociales Y Humanas, 17(32), 161-170.

doi:http://dx.doi.org/10.22518/16578953.824

Ricoeur, P. (2017). Hermenéutica. Escritos y conferencias 2. (A. Neira, Trad.) España: Editorial Trotta.

Rodríguez, R., Gómez, J., Rodríguez, J., Flórez, A., Quintero, M., Aguilar, A., \& Vivas, M. (2017). B-learning y didáctica para el trabajo colaborativo en la educación superior. En A. Laguado, A. García, \& U. D. Luz (Ed.), Las tecnologías de información y comunicación y la gestión empresarial (Vol. 1, págs. 408-426).

Torres, A., \& Romero, L. (2018). Gamificación en Iberoamérica: Experiencias desde la comunicación y la educación. Quito, Ecuador: Editorial Universitaria Abya-Yala. Obtenido de https://dspace.ups.edu.ec/bitstream/123456789/16425/4/Gamificacion\%20en\%20iberoamerica.pdf\#page $=192$ 
Visbal, R. (Julio - Diciembre de 2020). La lectura en espacios virtuales y digitales: un cambio social en el desarrollo del pensamiento crítico. Revista Neuronum, 6(2), 256-264. Obtenido de http://eduneuro.com/revista/index.php/revistaneuronum/article/view/257

Esta obra está bajo una Licencia Creative Commons Attribución-NoCommercial 4.0 International

(cc) EY-NG 